\title{
Novasorb® Cationic Nanoemulsion and Latanoprost: the Ideal Combination for Glaucoma Management?
}

\section{Philippe Daull, Mourad Amrane and Jean-Sébastien Garrigue*}

Santen SAS, Novagali Innovation Center, Evry, France

*Corresponding author: Jean-Sébastien Garrigue, Santen SAS 1, Rue Pierre Fontaine Bâtiment Genavenir IV, F-91058-Evry cedex, France, Tel: + 33-0-1-69-87-40-20; Fax: +33-0-1-69-87-40-30; E-mail: jean sebastien.garrigue@santen.fr

Received date: October 04, 2016; Accepted date: January 23, 2017; Published date: January 26, 2017

Copyright: (C) 2017 Daull P, et al. This is an open-access article distributed under the terms of the Creative Commons Attribution License, which permits unrestricted use, distribution, and reproduction in any medium, provided the original author and source are credited.

\section{Description}

Novasorb $^{\circledR}$ is a patented eye drop formulation platform developed to optimise the interaction of the eye drop-the cationic nanoemulsionwith the different layers of the tear film, mainly with the tear film lipid layer (TFLL), and the ocular surface [1]. The composition of the cationic nanoemulsion was designed to mimic the attributes and functions of the tear film and TFLL, and take advantage of the negatively-charged mucin layer covering the corneal and conjunctival epithelium [2], to increase its spreading and residence time on the ocular surface [3]. Consequently, Novasorb -based artificial tears (AT, e.g. Cationorm ${ }^{\circ}$ ) are functionally and mechanically very close to a healthy tear film; with an iso-osmolar to slightly hypo-osmolar aqueous phase, polar (cetalkonium chloride, CKC) and nonpolar (mineral oils or medium chain triglycerides, MCT) lipids, and surfactants (e.g. Tyloxapol and Poloxamer) that mimic the surface active proteins present at the interface with the TFLL. As a result, these cationic nanoemulsions interact favourably with Meibomian gland secretion films (i.e. TFLL) and enhance their structure and surface properties [4]. A stable TFLL is a key for the stabilization of a thin and uniform aqueous film over a convex structure like the eye [5] and protection of the corneal epithelium from desiccation and damages [6]. Indeed, a thin TFLL has been correlated with tear film instability (as measured by tear break-up time, TBUT)-the thinner the TFLL, the shorter the TBUT [7] and dry eye disease (DED) [8,9] or Meibomian gland dysfunction (MGD) [10,11].

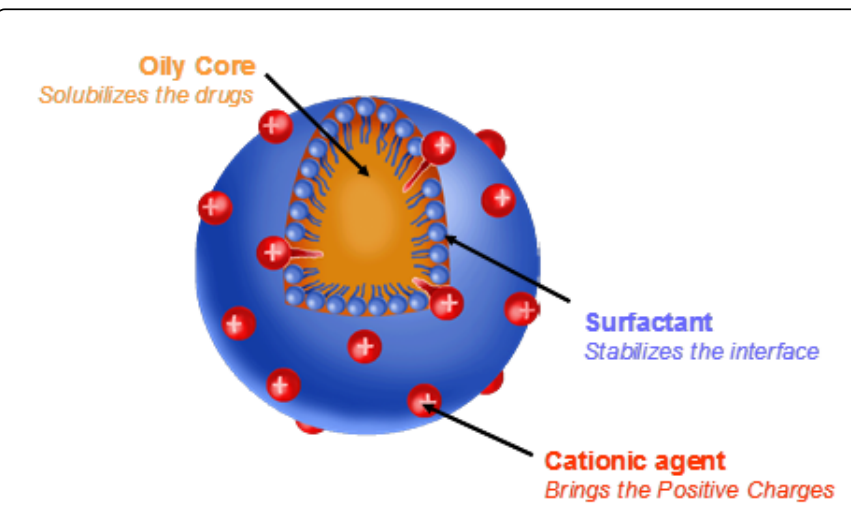

Figure 1: Schematic representation of an oil droplet from the cationic nanoemulsion.

In addition to the tear film stabilization properties brought by the cationic nanoemulsions (Figure 1), these eye drops also possess interesting wound healing [12] and anti-inflammatory properties $[13,14]$ that may contribute to the efficacy profile seen in DED patients. CKC, the cationic agent used in Novasorb ${ }^{\circledR}$ was demonstrated to be a specific protein kinase $\mathrm{C}$ alpha (PKCa) inhibitor [14]. Using PKCa-/- knockout mice, Chen and collaborators [15] have demonstrated the benefits of $\mathrm{PKC} \alpha$ modulation for corneal epithelial healing. $\mathrm{PKCa}-/-$ mice showed more rapid corneal healing, with less neutrophil infiltration, and a deficient neutrophil infiltration is one of the possible mechanisms for the accelerated corneal reepithelialization observed by Chen and collaborators in their study $[16,17]$. DED patients treated with the cationic nanoemulsions Cationorm ${ }^{\oplus}[18]$, or Ikervis ${ }^{\oplus}$ 's vehicle [19] experienced improvement of both their symptoms and signs of DED; with among them improved tear film properties (increased TBUT), and improved corneal fluorescein staining (CFS) with a rapid onset of improvement evident at 7 days [18]. These cationic nanoemulsions are also ideal to solubilize, in their oily phase, lipophilic drug substances such as cyclosporine A (CsA) or latanoprost. Indeed, the increased residence time of the cationic nanoemulsions on the ocular surface make them very good drug delivery vehicles for CsA [20] or latanoprost [21].

Glaucoma and DED signs and symptoms commonly occur together, and this overlap suggests an association between the two diseases. It is estimated that $50 \%$ to $60 \%$ of glaucoma patients have symptoms of ocular surface disease (OSD), e.g. keratitis, conjunctivitis and lid disease [22,23]. Abnormal TBUT with elevated Ocular Surface Disease Index (OSDI) and lissamine green scores were frequent in glaucoma patients when compared with controls [24]. OSD have been found to be aggravated by the use of preserved topical intraocular pressure (IOP)-lowering drugs $[25,26]$ and to play an important role in patient satisfaction and compliance. Indeed, the presence of OSD, hyperaemia, ocular signs and symptoms following instillation and the use of tear substitutes was statistically and significantly associated with patient dissatisfaction with their glaucoma treatment [27]. In addition, Batra and collaborators recently demonstrated that OSD management resulted in improved IOP control, suggesting that improving the ocular surface condition may help in the control of IOP [28].

Thus, in an attempt to improve glaucoma patients' quality-of-life and treatment compliance benzalkonium chloride (BAK)-free and soft-preserved eye drops were developed. While these new formulations were better tolerated, although recent studies have raised suspicion around long-term safety of other preservatives $[29,30]$ they do not protect or help in the restoration of the diseased ocular surface of glaucoma patients with OSD.

In that respect, the development of a preservative-free formulation of latanoprost (a very effective IOP-lowering prostaglandin analogue (PGA)) formulated in the Novasorb ${ }^{\star}$ technology, i.e., the cationic nanoemulsion AT vehicle which improves TFLL surface properties [4] and possesses wound healing and anti-inflammatory properties $[13,14]$ seems to be a reasonable approach. 


\section{Change in CFS at Day 10 (vs Day 3, before treatment)}

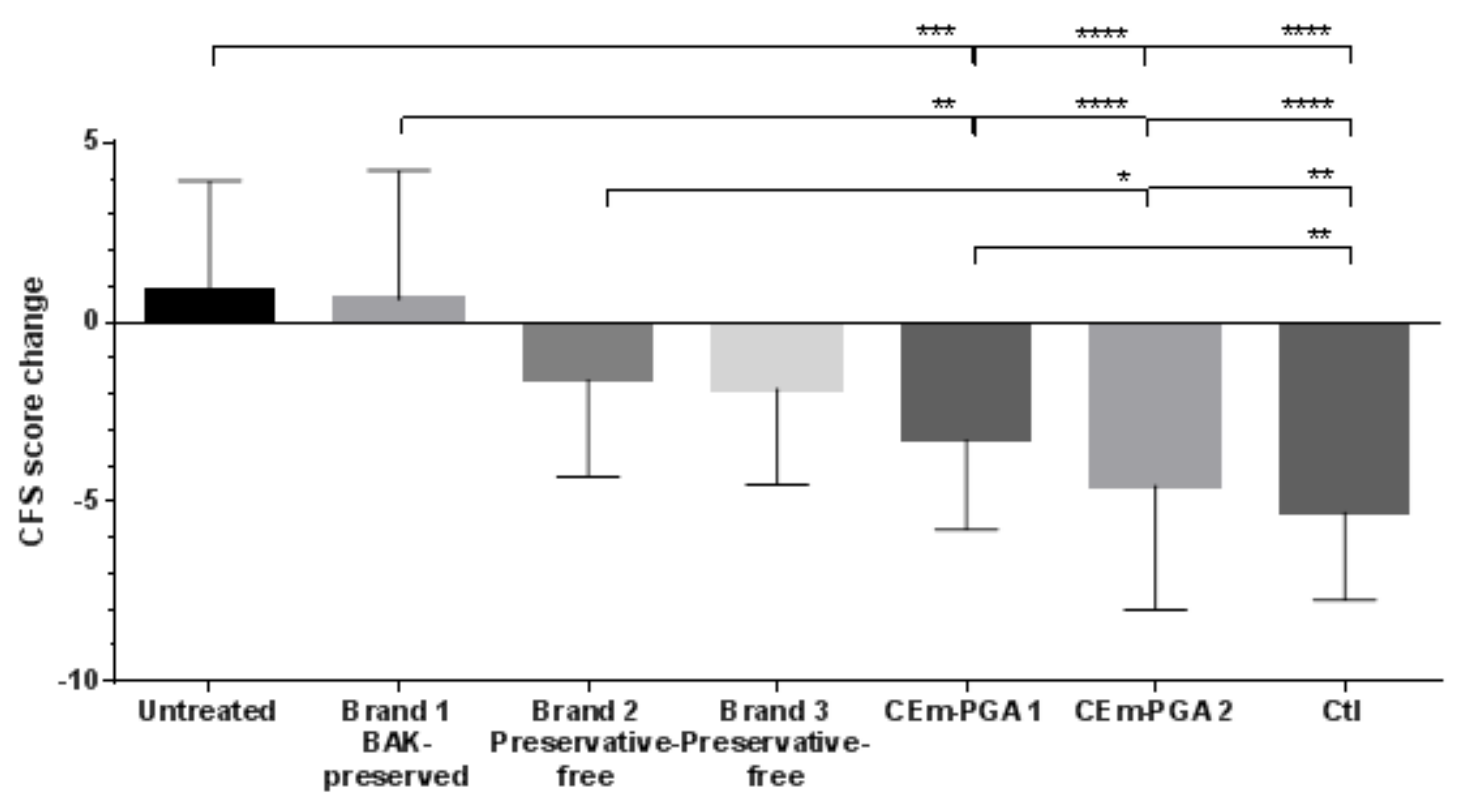

Figure 2: Mean reduction in CFS score at Day 10 (vs. Day 3, before treatment) following treatment with various formulations of PGAs IOPlowering drugs in the controlled environment mouse model of dry eye. Mice were placed in a controlled environmental chamber (CEC) for 10 days (temperature: $20-22^{\circ} \mathrm{C}$; relative humidity: $<25 \%$; airflow: $\left.15 \mathrm{~L} / \mathrm{min}\right)$, and treated with transdermal scopolamine patch administration $(0.5$ $\mathrm{mg} / 72 \mathrm{~h}$ ) on Day $1,3,5,7$ and 9 . The mice ( $\mathrm{n}=10$ per group) were then randomly assigned to 7 groups, including negative (untreated) and positive control groups. Three microliters of the test items were instilled three times a day in both eyes from Day 4 to 10 . The treatments were encoded, and the group allocation was blinded to the technician administering the treatment, and to the researcher assessing the outcome of the experiment. Group identification was uncovered at the end of the analysis. CFS (evaluated using the National Eye Institute scheme) was performed before dry eye induction (Day 0), and during the experiment at days 3,6 and 10 according to a previously published protocol 33 . Briefly, $0.5 \mu \mathrm{L}$ of a $0.5 \%$ fluorescein sodium solution was instilled into the inferior conjunctival sac using a micropipette. The cornea was examined through a biomicroscope by light passing through a cobalt blue filter. The stained area was assessed and graded using the grading system from the NEI/Industry Workshop guideline [38]. The system provided a stepwise categorization of the cornea, by dividing it into five sectors; with each one of them scored on a 0-3 scale, for a total maximal score of 15 . Statistical significance was set at a palue of 0.05 . Results are presented as mean $\pm \mathrm{SD}$.

Preclinical data demonstrated the good performance of PGAs cationic nanoemulsions in animal models with healthy or diseased ocular surface. Liang and collaborators have demonstrated with an acute toxicity rabbit model and in vivo confocal microscopy (IVCM) analysis of the cornea and conjunctiva associated lymphoid tissue that the cationic nanoemulsion of latanoprost was as well tolerated as a saline control. CD45 positive cells (i.e. inflammatory cells) count from conjunctival imprints were similar for the cationic nanoemulsion of latanoprost and PBS $(<10 \%)$, while with Xalatan ${ }^{\oplus}$, the CD45 positive cells represented more than $80 \%$ of the cells present on the imprints [31]. In addition, the cationic nanoemulsion of latanoprost was as effective as Xalatan ${ }^{\circ}$ in reducing IOP in a monkey model of elevated IOP, and was very well tolerated by the rabbit ocular surface, with a $42 \%$ reduction in hyperaemia incidence when compared to Xalatan ${ }^{\circledR}$ [21].

The effects of the cationic nanoemulsion of PGAs on damaged cornea were assessed in a rat model of corneal epithelial damage 12, or in a mouse model of dry eye. Following corneal scraping and a twice daily treatment for five days with either the cationic nanoemulsion of latanoprost, Xalatan', and their respective vehicles, the corneas were clinically evaluated (by IVCM). Treatments with the cationic nanoemulsion of latanoprost or its vehicle allowed for a complete and almost scar-free re-epithelisation of the cornea. Of particular notice, the remaining scar following treatment with the cationic nanoemulsion of latanoprost was approximately one third of the one following PBS treatment. By contrast, treatment with Xalatan ${ }^{\circledR}$ or its vehicle resulted in the formation of an important opaque scar underneath the healed epithelium [12]. This suggests that the cationic nanoemulsion of latanoprost was able to promote a safe healing process of the diseased cornea. The inflammatory cells count at the end of the treatment was also significantly lower following treatments with the cationic nanoemulsion of latanoprost and its vehicle when compared to Xalatan ${ }^{\bullet}$ [12]. In a mouse model of dry eye (controlled environment chamber mouse model of dry eye) [32-34] the PGA cationic nanoemulsions were able to reduce CFS score better than 
other preservative-free PGA formulations (Figure 2), and almost to the same extent than the positive control.

Phase II clinical data with the cationic nanoemulsion of latanoprost (Catioprost $^{\circledR}$ ) in glaucoma patients with OSD (with IOP at least 22 $\mathrm{mmHg}$ or higher, CFS score at least 1 or higher on the modified Oxford scale, ocular discomfort score at least 2 or higher on a $0-5$ symptom scale and TBUT equal or lower than 10 seconds) demonstrated that Catioprost $^{\bullet}$ was as effective as $\operatorname{Travatan}^{\circledR} \mathrm{Z}$ at lowering elevated IOP and decreasing the signs and symptoms of DED (Figure 3). Compared to $\operatorname{Travatan}^{\circ} \mathrm{Z}$, (a soft-preserved BAK-free PGA);
Catioprost $^{\oplus}$ reduced IOP to the same level than $\operatorname{Travatan}^{\oplus} \mathrm{Z}$ and induced less conjunctival hyperaemia ( $13 \%$ vs. $20 \%$ relative increase in conjunctival hyperaemia, respectively). At three months, in the full analysis set (FAS) population, Catioprost ${ }^{\oplus}$ group shows a reduction of CFS score of $24 \%$ compared to $9 \%$ in the Travatan ${ }^{\circ} \mathrm{Z}$ group (NS). The difference reached statistical significance in the per protocol (PP) population $(-30 \%$ vs. $-5 \%, \mathrm{p}<0.05$, respectively). In addition, Catioprost $^{\circ}$ was as good as $\operatorname{Travatan}^{\circ} \mathrm{Z}$ at improving TBUT $(40.2 \%$ vs. $36.9 \%$, respectively) and at reducing ocular discomfort symptoms (-48\% vs. $-43 \%$, respectively).
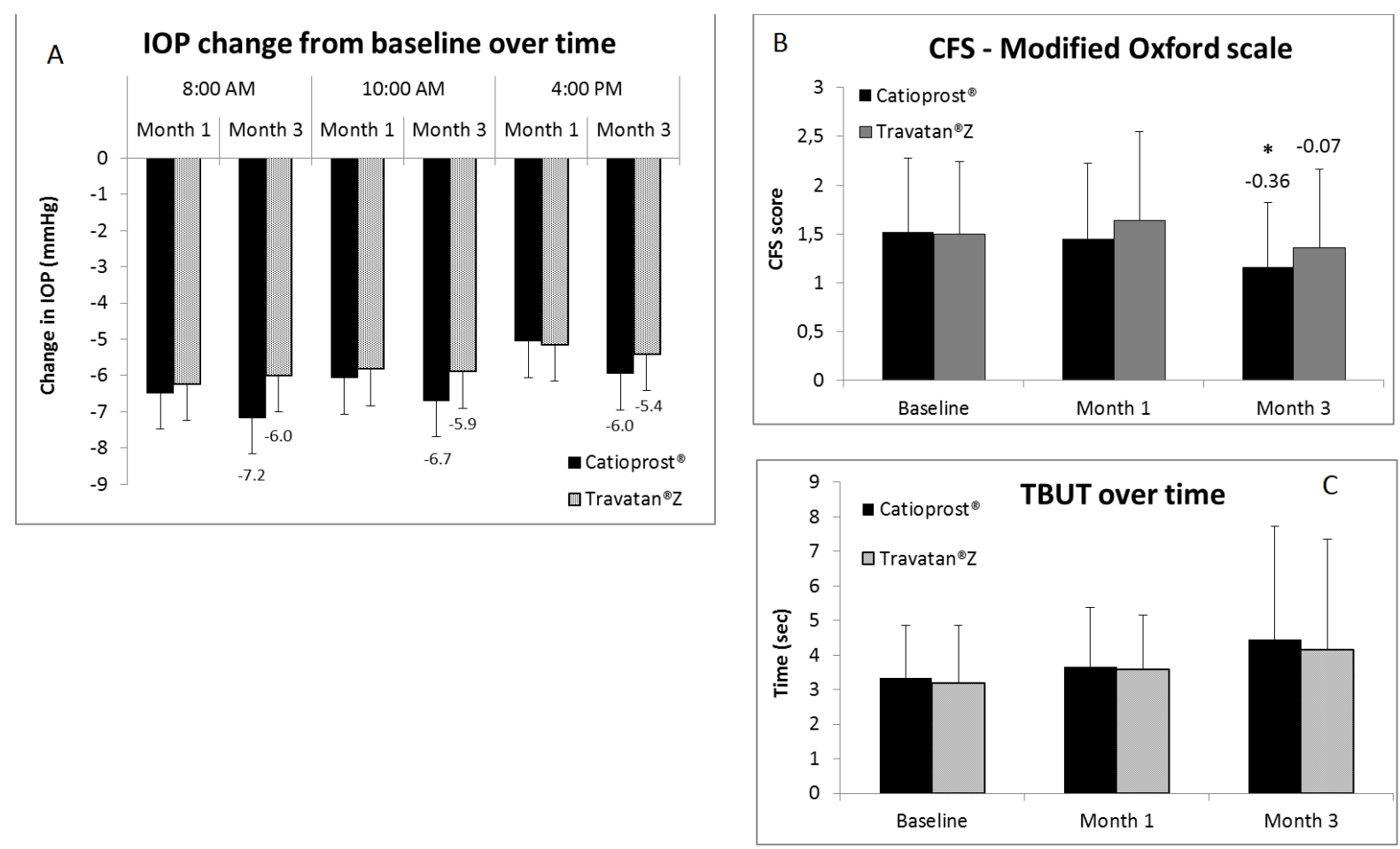

Figure 3: In patients treated with Catioprost ${ }^{\oplus}(n=51)$ or $\operatorname{Travatan}^{\circ} \mathrm{Z}(\mathrm{n}=54),(\mathrm{A})$ intraocular pressure reduction over time at 8:00 am, 10:00 am and 4:00 pm. Note the trend of a better IOP management at month 3 with Catioprost ${ }^{\circ}$. (B) Corneal fluorescein staining (CFS) score over time. At month 3 the change in CFS is indicated on the histogram bars. (C) Tear film break-up time (TBUT) at month $3^{*}, \mathrm{p}<0.05$ between both groups in the PP population. Briefly, this was a multicenter, phase 2, randomized, investigator-masked, active-control study in which subjects underwent a 2 to 5 week washout period for ocular hypertensive medications prior to baseline visit, followed by a 12-week dosing period with Catioprost $^{\oplus}$ or $\operatorname{Travatan}^{\oplus} \mathrm{Z}, \mathrm{QD}($ at $8 \mathrm{PM} \pm 1 \mathrm{~h}$ ). At screening, patients had to stop any ocular hypertensive medications and entered the washout phase during which all subjects were to receive AT (Refresh PlusTM, Allergan) to be used, if needed, as a relief medication for ocular discomfort. The mean inclusion criteria were IOP $\geq 22 \mathrm{mmHg}$, OSDI $\geq 2$ and CFS $\geq 1$, and TBUT $\mathrm{s} \leq 10$ seconds. In the FAS population, the mean subject age was 64.6 years (range, $41-88)$ and there were $34(32.4 \%)$ males and 71 (67.6\%) females, of whom 96.2\% were of nonHispanic/Latino ethnicity and of whom $60.0 \%$ were of White race and $39.0 \%$ were of Black or African American race. Among the study subjects, there were $48(22.9 \%)$ blue eyes, $138(65.6 \%)$ brown, $18(8.6 \%)$ hazel, and $6(2.9 \%)$ green. At baseline, the CFS strata was $\leq 1$ for 30 $(58.8 \%)$ and $33(61.1 \%)$ for Catioprost and TravatanZ, respectively and $>1$ for 21 (41.2\%) and 21 (38.9\%) for Catioprost and TravatanZ, respectively. Corneal pachymetry was done at screening to assess subjects' eligibility for inclusion in the study (subjects had to have a central corneal thickness (CCT) $\leq 620 \mu \mathrm{m}$ in each eye). Mean CCT was $554.6 \mu \mathrm{m}$ in the worst eyes and $555.5 \mu \mathrm{m}$ in the other eyes.

These clinical data are very promising and need to be confirmed in larger Phase III clinical trials. However, they suggest that the cationic nanoemulsion of latanoprost (Catioprost ${ }^{\circledR}$ ) has beneficial properties beyond the simple removal of BAK. By restoring and stabilising the tear film alongside with its anti-inflammatory and wound healing properties the cationic nanoemulsion contributes to the protection and restoration of the corneal epithelium $[35,36]$.

\section{Conclusion}

In conclusion, the cationic nanoemulsion of latanoprost is a very promising new eye drop formulation for the concomitant treatment of elevated IOP and OSD in glaucoma patients that goes beyond the simple 'ocular friendly' BAK-free aqueous solution anti-glaucoma drugs [37]. 


\section{References}

1. Lallemand F, Daull P, Benita S, Buggage R, Garrigue JS (2012) Successfully improving ocular drug delivery using the cationic nanoemulsion Novasorb. J Drug Deliv 2012: 604204.

2. Royle L, Matthews E, Corfield A, Berry M, Rudd PM (2008) Glycan structures of ocular surface mucins in man, rabbit and dog display species differences. Glycoconi J 25: 763-773.

3. Daull P, Lallemand F, Garrigue JS (2014) Benefits of cetalkonium chloride cationic oil-in-water nanoemulsions for topical ophthalmic drug delivery. J Pharm Pharmacol 66: 531-541.

4. Georgiev GA, Yokoi N, Ivanova S, Tonchev V, Daull P (2016) Surface chemistry of the interactions of cationic nanoemulsions with human meibum films. Acta Ophthalmologica 95.

5. Millar TJ, Schuett BS (2015) The real reason for having a meibomian lipid layer covering the outer surface of the tear film-A review Exp Eye Res.

6. Yokoi N, Bron AJ, Georgiev GA (2014) The precorneal tear film as a fluid shell: the effect of blinking and saccades on tear film distribution and dynamics. Ocul Surf 12: 252-266.

7. Isreb MA, Greiner JV, Korb DR, Glonek T, Mody SS (2003) Correlation of lipid layer thickness measurements with fluorescein tear film break-up time and Schirmer's test. Eye 17: 79-83.

8. Yokoi N, Takehisa Y, Kinoshita S (1996) Correlation of tear lipid layer interference patterns with the diagnosis and severity of dry eye. Am Ophthalmol 122: 818-824.

9. Foulks GN (2007) The correlation between the tear film lipid layer and dry eye disease. Surv Ophthalmol 52: 369-374.

10. Eom Y, Lee JS, Kang SY, Kim HM, Song JS (2013) Correlation Between Quantitative Measurements of Tear Film Lipid Layer Thickness and Meibomian Gland Loss in Patients With Obstructive Meibomian Gland Dysfunction and Normal Controls. Am J Ophthalmol 155: 1104-1110.e2.

11. Finis D, Pischel N, Schrader S, Geerling G (2013) Evaluation of lipid layer thickness measurement of the tear film as a diagnostic tool for Meibomian gland dysfunction. Cornea 32: 1549-1553.

12. Liang H, Baudouin C, Daull P, Garrigue JS, Brignole-Baudouin F (2012) In vitro and in vivo evaluation of a preservative-free cationic emulsion of latanoprost in corneal wound healing models. Cornea 31: 1319-1329.

13. Daull P, Feraille L, Elena PP, Garrigue JS (2016) Comparison of the AntiInflammatory Effects of Artificial Tears in a Rat Model of Corneal Scraping. J Ocul Pharmacol Ther 32: 109-118.

14. Daull P, Guenin S, Garrigue JS (2016) Mechanism of action of cationic emulsions in the management of ocular surface inflammation and wound healing. ARVO. Seattle.

15. Chen Z, Li Z, Basti S, Farley WJ, Pflugfelder SC (2010) Altered morphology and function of the lacrimal functional unit in protein kinase C\{alpha\} knockout mice. Invest Ophthalmol Vis Sci 51: 5592-5600.

16. Li Z, Burns AR, Smith CW (2006) Lymphocyte function-associated antigen-1-dependent inhibition of corneal wound healing. Am J Pathol 169: $1590-1600$

17. Ueno M, Lyons BL, Burzenski LM, Gott B, Shaffer DJ, et al. (2005) Accelerated wound healing of alkali-burned corneas in MRL mice is associated with a reduced inflammatory signature. Invest Ophthalmol Vis Sci 46: 4097-4106.

18. Robert P-Y, Cochener B, Amrane M, Ismail D, Garrigue JS, et al. (2016) Efficacy and safety of a cationic emulsion in the treatment of moderate to severe dry eye disease: a randomized controlled study. Eur J Ophthalmol 26: 546-555.

19. Leonardi A, Van Setten G, Amrane M, Ismail D, Garrigue JS, et al. (2016) Efficacy and safety of $0.1 \%$ cyclosporine A cationic emulsion in the treatment of severe dry eye disease: a multicenter randomized trial. Eur J Ophthalmol 26: 287-296.
20. Daull P, Lallemand F, Philips B, Lambert G, Buggage R, et al. (2013) Distribution of cyclosporine $\mathrm{A}$ in ocular tissues after topical administration of cyclosporine A cationic emulsions to pigmented rabbits. Cornea 32: 345-354.

21. Daull P, Buggage R, Lambert G, Faure MO, Serle J, et al. (2012) A comparative study of a preservative-free Latanoprost cationic emulsion (Catioprost) and a BAK-preserved Latanoprost solution in animal models. JOPT 28: 515-523.

22. Leung EW, Medeiros FA, Weinreb RN (2008) Prevalence of ocular surface disease in glaucoma patients. J Glaucoma 17: 350-355.

23. Fechtner RD, Godfrey DG, Budenz D, Stewart JA, Stewart WC, et al. (2010) Prevalence of Ocular Surface Complaints in Patients With Glaucoma Using Topical Intraocular Pressure-Lowering Medications. Cornea 29: 618-621.

24. Saade CE, Lari HB, Berezina TL, Fechtner RD, Khouri AS (2015) Topical glaucoma therapy and ocular surface disease: a prospective, controlled cohort study. Can J Ophthalmol 50: 132-136.

25. Pisella PJ, Pouliquen P, Baudouin C (2002) Prevalence of ocular symptoms and signs with preserved and preservative free glaucoma medication. Br J Ophthalmol 86: 418-423.

26. Baudouin C, Labbe A, Liang H, Pauly A, Brignole-Baudouin F (2010) Preservatives in eyedrops: the good, the bad and the ugly. Prog Retin Eye Res 29: 312-334.

27. Lemij HG, Hoevenaars JG, van der Windt C, Baudouin C (2015) Patient satisfaction with glaucoma therapy: reality or myth? Clin Ophthalmol 9: 785-793.

28. Batra R, Tailor R, Mohamed S (2014) Ocular surface disease exacerbated glaucoma: optimizing the ocular surface improves intraocular pressure control. J Glaucoma 23: 56-60.

29. Gandolfi S, Paredes T, Goldberg I, Coote M, Wells A, et al. (2012) Comparison of a travoprost BAK-free formulation preserved with polyquaternium-1 with BAK-preserved travoprost in ocular hypertension or open-angle glaucoma. Eur J Ophthalmol 22: 34-44.

30. Katz G, Springs CL, Craven ER, Montecchi-Palmer M (2010) Ocular surface disease in patients with glaucoma or ocular hypertension treated with either BAK-preserved latanoprost or BAK-free travoprost. Clin Ophthalmol 4: 1253-1261.

31. Liang H, Baudouin C, Faure MO, Lambert G, Brignole-Baudouin F (2009) Comparison of the ocular tolerability of a latanoprost cationic emulsion versus conventional formulations of prostaglandins: an in vivo toxicity assay. Mol Vis 15: 1690-16999.

32. Barabino S, Shen L, Chen L, Rashid S, Rolando M, et al. (2005) The controlled-environment chamber: a new mouse model of dry eye. Invest Ophthalmol Vis Sci 46: 2766-2771.

33. Barabino S, Antonelli S, Cimbolini N, Mauro V, Bouzin M (2014) The effect of preservatives and antiglaucoma treatments on the ocular surface of mice with dry eye. Invest Ophthalmol Vis Sci 55: 6499-6504.

34. Daull P, Feraille L, Barabino S, Cimbolini N, Antonelli S, et al. (2016) Efficacy of a new topical cationic emulsion of cyclosporine A on dry eye clinical signs in an experimental mouse model of dry eye. Exp Eye Res 153: 159-164.

35. Mundorf TK (2011) Treat glaucoma patients globally. Adv Ocular Care 71-72.

36. Godfrey DA (2011) Glaucoma: What's next? Ophthalmology Times $42-43$

37. Abelson MB, Lafond A (2011) Glaucoma and Dry Eye: A Tough Combo. Rev Ophthal :108-111.

38. Lemp MA (1995) Report of the National Eye Institute/Industry workshop on Clinical Trials in Dry Eyes. CLAO J 21: 221-232. 